\title{
Aris-Taylor dispersion in the subarachnoid space
}

\author{
Luca Salerno $\odot,^{*}$ Giulia Cardillo $\odot,^{\dagger}$ and Carlo Camporeale $\left(^{\ddagger}\right.$ \\ Politecnico di Torino, Corso Duca degli Abruzzi 24, 10129 Torino, Italy ${ }^{\S}$
}

(Received 23 January 2019; accepted 16 March 2020; published 27 April 2020)

\begin{abstract}
A complete theory able to assess the longitudinal dispersion of a passive solute injected into an annular cavity subject to a pulsatile flow and a porous medium is provided. The Aris-Taylor method of statistical moments is combined with the Brinkman approach for porous flows and morphological dispersion, in order to get an analytical relationship for the time-dependent enhanced dispersion coefficient. The application of intrathecal drug delivery in the cerebrospinal fluid contained in the subaracnoid space is discussed in detail. The main result of the theory and its assumptions are also numerically validated through the use of a finite-volume solver. The role of several physiological features, such as the geometry, temporal frequency, and wavelength, of the pressure forcing are analyzed. It turns out that the presence of delicate strands of connective tissue, called trabeculae, that fill the cavity and link the innermost layer of meninges plays a crucial role. They in fact induce extra terms of morphological dispersion and act synergistically with pulsation to produce realistic times of drug delivery in clinically significant conditions. The results have potential for the optimization of delivery protocols of drug therapies of the central nervous system.
\end{abstract}

DOI: 10.1103/PhysRevFluids.5.043102

\section{INTRODUCTION}

When an inert solute is introduced into a Poiseuille flow, the combined effect of random lateral excursions caused by diffusion and the velocity profile causes an enhanced longitudinal dispersion. If the flow regime is pulsatile, differential advection and transversal diffusion combine in order to cause in each cycle a progressive longitudinal spreading of solute having an effective diffusivity much larger than the molecular one. More specifically, the initially uniform plug of solute is stretched by shear flow, so that the particles moving at different speeds increase their relative distance, generating concentration gradients in the transverse direction. Such gradients promote transverse diffusion, which dislocates particles from one streamline to another with a different speed and concentration. This mixing assures that during backflow the particle may not return to its original position, causing a net longitudinal spread in each cycle. The result of this phenomenon is shown in Fig. 1.

If the transverse section is not small enough to be completely affected by diffusion within one cycle $\left(2 \pi / \omega^{*} \gg \delta_{r}^{* 2} / 4 \mathcal{D}\right)$, an elevated number of oscillations is needed before a particle has moved within the whole range of velocity. So, for small times $\left(t<\omega^{-1}\right)$ the process is mainly regulated by molecular diffusion, while for longer times the solute spreads with a shear-enhanced diffusivity.

Shear-flow-enhanced dispersion was first analyzed by Taylor [1], who provided a complete description of the asymptotic, cross-sectionally averaged axial concentration distribution under steady-flow conditions. The analysis was extended to a wider range of Péclet numbers and

\footnotetext{
*1uca.salerno@polito.it

† giulia.cardillo@polito.it

¥carlo.camporeale@polito.it

$\S_{\text {http://www.envirofluidgroup.it }}$
} 


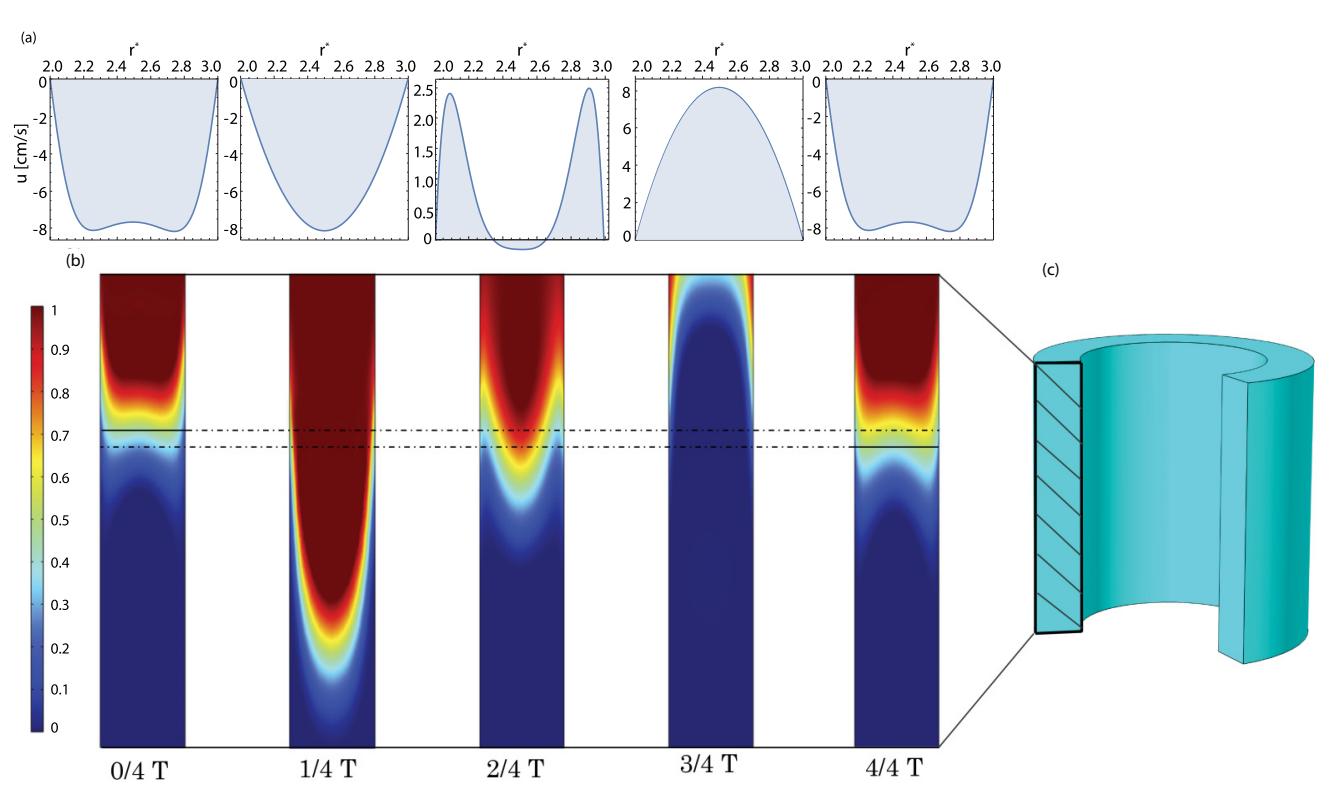

FIG. 1. Numerical solution of the tracer dispersion in the time span of a single period (see Appendix B). (a) Lateral distribution of CSF velocity in the SAS at five times. (b) Longitudinal view of the concentration field (the injection point is located at the top of the panel). The solid portions of the horizontal lines mark the front of the same isoconcentration contour line $(c=0.6)$ at times $t$ and $t+T$, respectively. The advance of the front is evident. (c) Three-dimensional view of the computational domain.

geometries by Aris [2] using the method of statistical moments. The Taylor-Aris formulation is limited to the case of steady flows but Watson [3] evaluated the increase in mass rate transfer of a diffusing substance for oscillatory flows in circular pipes. Results were given for a general cross section in the limiting cases of slow and fast oscillations of the flow. Vedel and Bruus [4,5] combined the Aris method of moments with Dirac's bra-ket formalism and obtained a relation for the effective solute diffusivity $D_{e}(t)$, for a given time-dependent laminar flow, in a straight channel of a generic cross-sectional shape. The aim of the present study is to develop a self-consistent analytical dispersion theory tailored for a drug injection into the spinal subarachnoid space, wherein the cerebrospinal fluid (CSF) has a pulsatile flow, through an extension of the Vedel and Bruus [4] formulation to porous media.

The cerebrospinal fluid is a Newtonian fluid, with a density and kinematic viscosity similar to those of water [6,7], which is continuously secreted from the blood plasma in the choroid plexus of the brain ventricles [6]. Its primary mechanical function is to protect the brain and the spinal cord, serving as a shock absorber for the central nervous system [8]. It allows changes in intracranial pressure to be modulated, and along with the cerebral blood flow, it regulates the intracranial pressure itself [9]. The CSF fills the subarachnoid space (SAS), a thin annular cavity surrounding the spinal cord, where the CSF is subject to a pulsatile laminar flow essentially driven by changes in the intracranial blood volume during the cardiac cycle [7,9] and modulated by other physiological factors, such as the respiratory rate [10]. Intrathecal drug delivery is the administration of medications into the subarachnoid space through injection. This procedure is currently used in clinical practice and it is promising for the treatment of several pathologies of the central nervous system (including some cancers [11] and CSF infection [12]) and for the administration of analgesic drugs that cannot be delivered systematically [13]. However, intrathecal delivery protocols and facilities are not optimized yet, since under- or overdosage frequently occurs, thus affecting the anticancer effect or inducing nerve damage $[14,15]$. Therefore the fluid dynamic processes and the effective diffusivity of these medications urgently need to be assessed quantitatively. 
In the literature, there are important experimental and computational studies evaluating the dispersion of drugs in the SAS. Hettiarachchi et al. [13] conducted infusion tests with a radionucleotide and fluorescent dye under both stagnant and pulsatile flow conditions, within an experimental surrogate model of the human spinal canal. Nelissen [10] developed a laser scanning setup with which he performed quasi-instantaneous, three-dimensional laser-induced fluorescence as well as two-dimensional particle image velocimetry. Hsu et al. [16] proposed a novel method, namely, a medical-image-based computational fluid dynamics, for investigating intrathecal drug delivery. This methodology combines quantitative medical imaging and CFD to generate patient-specific computational models. Haga et al. [17] investigated the effects of injection parameters on solute distribution within the cervical subarachnoid space, using a Lagrangian approach in a numerical platform. Pizzichelli et al. [18] introduced a numerical model able to simultaneously account for solute transport in the fluid and in the spinal cord, using a discontinuous Galerkin method and a three-dimensional patient-specific geometry.

Despite these valuable studies, a robust analytical model is still lacking. In this paper, we provide a consistent theory for the computation of the time-dependent dispersion coefficient, including the geometrical effects, the role of unsteadiness, and the important influence of anatomical fine network within the SAS. These fine structures within the SAS influence the transversal mixing and the CSF flow field, thus affecting the diffusion process.

Along with the analytical results, a numerical analysis is performed using software (Comsol) solving the equations for the momentum and solute transport. In this way, the influence of dispersion on the kinematic behavior of velocity waves (driven by pressure waves) is tested and the validity of theoretical results is discussed in detail. Wetheoretically show that shear, pulsatility, and porosity act in a synergistic way to enhance the dispersion of several orders of magnitude $[13,19]$ with respect to the pure molecular diffusion, a mechanism that turns out to be decisive in optimizing drug release and timing in pharmacological therapies of the nervous system, particularly when the target of the therapy is the brain.

The paper is organized as follows: Sec. II shows the mathematical problem, with a solution of the velocity field and computation of the dispersion coefficient; Sec. III provides a comparison between numerical and theoretical results and a sensitivity analysis and discusses the role of the internal layout. Some conclusions are drawn in Sec. IV. Finally, the nomenclature used is reported in Sec. V.

\section{ANALYTICAL MODEL}

The proposed analytical model evaluates the shear-induced enhanced drug diffusion in the flow direction. In particular, the role of convective transport associated with the pulsatile CSF flow is analyzed in the SAS. The latter is sketched as an annular cavity bounded by two concentric cylindrical surfaces, having radii equal to $r_{i}^{*}$ and $r_{e}^{*}$, respectively [see Fig. 2(c)]. In order to analyze the influence of the pulsatile flow and the fine structures within the SAS on the drug transport phenomenon, many anatomical details are deliberately simplified in the geometry. However, as we discuss in Sec. IV, the values of the CSF flow field are comparable to MRI measurements and to other SAS models $[6,7,20,21]$.

\section{A. Solution of the flow field}

The driving force of the flow can be divided into an unsteady oscillatory component inducing zero net flow and a constant component associated with steady streaming. The latter component is related to the production and reabsorption of the fluid in the brain. Under normal conditions, CSF is in fact produced in the ventricular cavity at the rate of $0.3 \div 0.7 \mathrm{ml} / \mathrm{min}$ [7], and it is reabsorbed into the arachnoid villi inside the cerebral ventricles. The magnitude of the CSF eliminated by the spinal canal is instead just conjectured since it is very small and undetectable in CINE-MRI measurements $[6,7,20,22]$. The magnitude of the steady streaming is therefore much smaller than the oscillatory component, which is caused by the pulse waves generated inside the rigid cranial vault [7] (during each systole 1-2 $\mathrm{ml}$ of CSF is pumped into the spinal SAS and returns to the cranial SAS during 
(a)

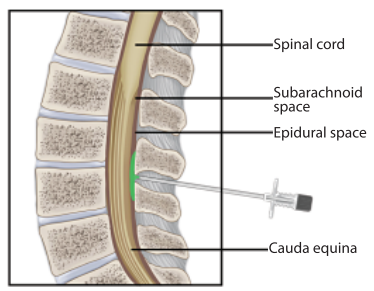

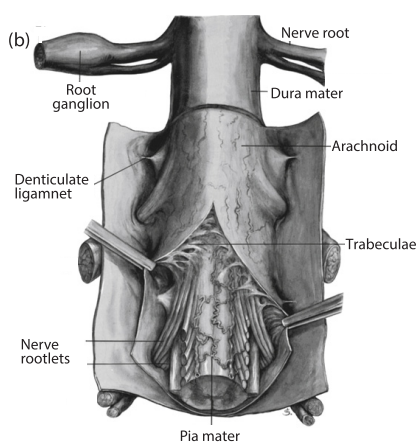

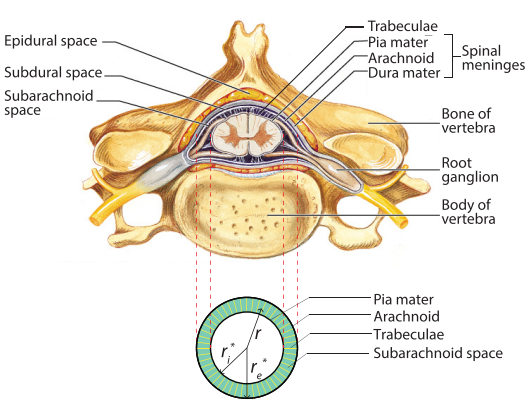

(c)

FIG. 2. Subarachnoid space and spinal cord. (a) Sagittal view of the spinal cord and intrathecal drug delivery. (b) Anatomical representation of the spinal cord and meninges. (c) Transversal section and model geometry.

dystole [6]). For this reason the steady streaming component will be neglected and we simply set

$$
\frac{\partial p^{*}}{\partial z^{*}}=\Delta^{*} \exp \left[\mathrm{i} \omega^{*} t^{*}\right]+\text { c.c. },
$$

where $\omega^{*}$ is the frequency of the CSF flow oscillation and $\Delta^{*}$ is the amplitude of the pulse pressure gradient. Henceforth, an asterisk indicates a nondimensionless quantity, while c.c. refers to complex conjugation.

After assuming axial symmetry and using the scales $\delta_{r}^{*}=r_{e}^{*}-r_{i}^{*}, L^{*}, U^{*}=U_{\max }^{*}, \mu U^{*} L^{*} / \delta_{r}^{* 2}$, and $2 \pi / \omega^{*}$ to nondimensionalize the radial and longitudinal lengths, velocities, pressures, and time, respectively (where $\mathcal{D}$ is the drug molecular diffusion, $\rho$ the fluid density, $\mu$ its dynamic viscosity, and $U_{\max }^{*}$ the maximum value of velocity), the longitudinal component of the Navier-Stokes equation reads

$$
\frac{W^{2}}{2 \pi} \frac{\partial u}{\partial t}+\frac{R_{e}}{n} u \frac{\partial u}{\partial z}+\frac{\partial p}{\partial z}=\frac{\partial^{2} u}{\partial r^{2}}+\frac{1}{r} \frac{\partial u}{\partial r}
$$

where $r$ and $z$ are the (dimensionless) radial and longitudinal coordinates, respectively, $p$ is the pressure, $u(r, z, t)$ is the longitudinal velocity, $R_{e}=\rho U^{*} \delta_{r}^{*} / \mu$ is the Reynolds number, $W=\sqrt{\omega^{*} \delta_{r}^{* 2} / v}$ is the Womersley number, namely, the ratio between the transient inertial forces and viscous forces, and $n=L^{*} / \delta_{r}^{*}$. If the temporal frequency of the oscillation is small (i.e., small Womersley number), the velocity has enough time to adapt to the change in the pressure gradient. Thus, the velocity changes almost in phase with the pressure gradient and its profile reduces to the Poiseuille flow driven by a constant pressure. On the contrary, if the oscillations are rapid (high Womersley number), there is a lag between velocity and pressure and the velocity distribution differs from the Poiseuille solution.

It is noteworthy that the physiological elasticity of the meninges induces pressure and velocity waves propagating with a celerity of about $5-10 \mathrm{~m} / \mathrm{s}$ and a wavelength of about $5-10 \mathrm{~m} \mathrm{[6].} \mathrm{Due}$ to the relative shortness of the spinal length $(\sim 0.5 \mathrm{~m})$ this celerity is high enough to assume that the velocity profile changes almost simultaneously throughout the spine in response to the pressure change. Additionally, under physiological conditions the quantities $W^{2} / 2 \pi$ and Re are comparable, and the length-to-gap ratio $n$ is about 100, so the second term in (2) can be safely neglected. These considerations allow us to neglect the wave nature of the flow fields, as if the boundaries were undeformable. Therefore, we are authorized to adopt the ansatz $u=\hat{u}(r) e^{i \omega t}$, make use of (1) in 
Eq. (2), and impose no-slip conditions on the walls $\left(r=r_{i}\right.$ and $\left.r=r_{i}+1\right)$, so obtaining

$$
\begin{gathered}
\frac{\partial^{2} \hat{u}}{\partial r^{2}}+\frac{1}{r} \frac{\partial \hat{u}}{\partial r}-\mathrm{i} W^{2} \hat{u}=\delta, \\
\hat{u}\left(r_{i}\right)=\hat{u}\left(r_{i}+1\right)=0,
\end{gathered}
$$

where $r_{i}=r_{i}^{*} / \delta_{r}^{*}$ and $\delta=\Delta^{*} \delta_{r}^{* 2} / U^{*} \mu$. The exact solution of problems (3) and (4) is

$$
\hat{u}(r)=u_{0}\left\{1-b_{1} J_{0}[\alpha r]+b_{2} Y_{0}[\alpha r]\right\},
$$

where $\alpha=\mathrm{i}^{3 / 2} W, u_{0}=\delta / \alpha^{2}, J_{0}$ and $Y_{0}$ are Bessel functions of order 0 of the first and second kinds, respectively, and $b_{1}$ and $b_{2}$ fulfill the boundary conditions, (4), and read

$$
\begin{aligned}
b_{1} & =\frac{Y_{0}\left[-\alpha-\alpha r_{i}\right]-Y_{0}\left[-\alpha r_{i}\right]}{J_{0}\left[\alpha r_{i}\right] Y_{0}\left[-\alpha-\alpha r_{i}\right]-J_{0}\left[\alpha+\alpha r_{i}\right] Y_{0}\left[-\alpha r_{i}\right]}, \\
b_{2} & =\frac{J_{0}\left[\alpha+\alpha r_{i}\right]-J_{0}\left[\alpha r_{i}\right]}{J_{0}\left[\alpha r_{i}\right] Y_{0}\left[-\alpha-\alpha r_{i}\right]-J_{0}\left[\alpha+\alpha r_{i}\right] Y_{0}\left[-\alpha r_{i}\right]} .
\end{aligned}
$$

\section{B. Role of porosity}

The internal layout of anatomical fine structures within the SAS is quite tangled. There are different types of structures (trabeculae, nerve roots, and ligaments), having specific physiological functions, which are characterized by different shapes, numbers, and sizes (see Fig. 2). In the present work, we focus on the obstruction caused by trabeculae, delicate strands of connective tissue linking the two innermost layers of the meninges. With the aim of taking into account these anatomical fine structures inside the SAS, the above modeling of the flow field is improved by adding a Brinkman term, $-u / \kappa$, to the right-hand side of the Navier Stokes equation [23],

$$
\frac{W^{2}}{2 \pi \phi} \frac{\partial u}{\partial t}+\frac{R_{e}}{n} u \frac{\partial u}{\partial z}+\frac{\partial p}{\partial z}=\frac{\partial^{2} u}{\partial r^{2}}+\frac{1}{r} \frac{\partial u}{\partial r}-\frac{u}{\kappa} .
$$

This term accounts for the momentum loss due to the porous medium, which is characterized by porosity $\phi$ (i.e., the ratio between the volume not filled by trabeculae $V_{\mathrm{tr}}^{*}$ and the total subarachnoid cavity volume) and permeability $\kappa^{*}=\delta_{r}^{* 2} \kappa$. This requires that in the following $u$ is intended as the local value of the volume-averaged velocity in the voids [24].

Trabeculae are randomly arranged structures, so in order to allow for an estimation of $\kappa^{*}$ and $\phi$, we consider a simplified layout made up of isotropic straight cylindrical elements connecting the meninges. We observe that a comparison performed among different approaches (Gupta et al. [23], Koch and Brady [25], Spielman and Goren [26]) to evaluate the permeability of a low-density fiber bed shows comparable results. Nevertheless, the formulation developed by Gupta et al. is also true when trabeculae are randomly arranged and it was tested for SAS anatomy [23]. The latter approach was based on a closed-form solution of the permeability of unidirectional fiber beds derived from phase-averaging of Navier-Stokes equations over a representative repetitive unit. In this way, a formula for the dimensionless permeability $\kappa=\kappa^{*} / \delta_{r}^{* 2}$ was obtained [27]:

$$
\kappa=\frac{\pi \phi(1-\sqrt{(1-\phi})^{2} r_{t}^{2}}{24(1-\phi)^{3 / 2}}
$$

where $r_{t}=r_{t}^{*} / \delta_{r}^{*}$ is the dimensionless trabecular radius.

After taking into account the above improvements, expression (5) is still a solution of Eq. (8) provided that $W$ is substituted by the quantity

$$
W_{p}=\sqrt{\frac{W^{2}}{\phi}-\frac{i}{\kappa}}
$$

Note that the term $i / \kappa$ induces a further phase lag between velocity and pressure. 


\section{Modeling of the dispersion}

Transport of solutes within the SAS is influenced by molecular diffusion, advection associated with the pulsatile CSF flow and the presence of anatomical fine structures within the cavity. In particular, the latter aspect induces a morphological dispersion due to different randomly distributed pathways that particles follow inside the porous matrix. The process of morphological dispersion has a long tradition in the fluid mechanics of porous media [25,26,28-30] and can be readily incorporated into the present analysis by considering the effective drug diffusivity as the sum of the molecular diffusivity and a $2 \times 2$ tensor $\mathbf{D}$, having $D_{L}$ and $D_{r}$ as the diagonal components (the longitudinal and radial directions, respectively) and 0 as the off-diagonal terms. Following Koch and Brady [25], $D_{L}$ and $D_{r}$ read

$$
D_{L} \sim \mathcal{D}\left[\frac{171}{3200} \pi^{3} \frac{r_{t}^{*}}{\sqrt{\kappa} \phi_{r}} P+\frac{243}{50} \frac{\phi_{r}^{2} r_{t}^{* 2}}{\kappa} P(\ln P)^{2}\right], \quad D_{r} \sim \frac{9}{6400} \frac{\pi^{3} r_{t}^{*} P}{\phi_{r} \sqrt{\kappa}} \mathcal{D},
$$

with

$$
\phi_{r}=1-\phi, \quad P=\frac{\hat{u}_{p} r_{t}^{*}}{\mathcal{D}},
$$

where $\phi_{r}$ is the volume fraction of trabeculae, $\hat{u}_{p}$ is the mean velocity of the fluid (the interstitial velocity), and $P$ is the Péclet number based on the trabecular radius.

In order to study this contribution, some assumptions are necessary: (i) the matrix is assumed to be rigid, impermeable, isotropic, and not influenced by CSF flow; (ii) the solute is considered as an inert scalar; (iii) drug absorption is neglected; and (iv) the solute concentration is sufficiently low, therefore it does not influence CSF properties. Recalling the above-mentioned scaling and introducing $C_{0}$ as the average drug concentration value, the dimensionless volume-averaged concentration in the voids $c(z, r, t)$ is driven by a standard advection-diffusion equation,

$$
\frac{\partial c}{\partial t}+(1+\gamma) \Omega^{2} u \frac{\partial c}{\partial z}=g_{L} \frac{\partial^{2} c}{\partial z^{2}}+g_{r} \frac{1}{r} \frac{\partial}{\partial r}\left(r \frac{\partial c}{\partial r}\right)
$$

where

$$
\Omega=\frac{\tau_{\omega}}{\tau_{r}}, \quad \gamma=\frac{1-\phi}{\phi}, \quad g_{L}=\frac{\tau_{\omega}}{\tau_{D L}}, \quad g_{r}=\frac{\tau_{\omega}}{\tau_{D r}}
$$

with

$$
\tau_{\omega}=\frac{2 \pi}{\omega^{*}}, \quad \tau_{r}=\frac{\delta_{r}^{*}}{U^{*}}, \quad \tau_{D L}=\frac{\delta_{r}^{* 2}}{\mathcal{D}+D_{L}}, \quad \tau_{D r}=\frac{\delta_{r}^{* 2}}{\mathcal{D}+D_{r}},
$$

representing the oscillating, shear, lateral diffusion, and transversal diffusion time scales, respectively.

The initial and boundary conditions associated with (13) are

$$
\begin{gathered}
\qquad(z, r, 0)=\tilde{c}(z, r), \\
\frac{\partial c}{\partial r}=0 \quad \text { for } \quad r=r_{i} \quad \text { and } r=1, \\
z^{a} \partial_{z}^{b} c \rightarrow 0 \text { for } z \rightarrow \frac{L}{2} \quad \text { and } \quad a, b=0,1,2 \ldots,
\end{gathered}
$$

where $\tilde{c}$ is the initial concentration field, Eq. (17) sets zero flux at the walls, and Eq. (18) sets smoothness of the solution at the upstream and downstream ends of the channel, $L$ being the dimensionless length of the cavity.

Following Aris's method, one has

$$
\frac{D_{e}^{*}}{\left(D_{L}+\mathcal{D}\right) / g_{L}}=\frac{1}{2} \frac{d \mu_{2}}{d t}=\frac{1}{2} \frac{d}{d t}\left(m_{2}-m_{1}^{2}\right),
$$


where $m_{p}$ is the $p$ th full moment of the solute concentration field, namely,

$$
m_{p}=\left\langle 1, c_{p}(r, t)\right\rangle=\left\langle 1, \frac{1}{L} \int_{-L / 2}^{L / 2} x^{p} c(r, z, t) d z\right\rangle,
$$

in which $c_{p}$ is the axial moment of concentration and the angular bracket notation refers to the internal product, defined by

$$
\langle f, g\rangle=\frac{2 \pi}{a} \int_{r_{i}}^{r_{i}+1} r f(r) g(r) d r,
$$

where $a$ is the cross section of the annular cavity.

Following the approach of Vedel and Bruus [4] for harmonic oscillating flow (an extension of Barton's [31] method) and including the morphological dispersion, we multiply (13) by $z^{p}$, integrate over $z$, and use (20). In this way, we get the recursive equations

$$
\begin{gathered}
\frac{\partial c_{p}}{\partial t}-\frac{g_{r}}{r} \frac{\partial}{\partial r}\left(r \frac{\partial c_{p}}{\partial r}\right)=g_{L} p(p-1) c_{p-2}(r, t)+\Omega(1+\gamma) u(r, t) p c_{p-1}(r, t), \\
\frac{d m_{p}(t)}{d t}=g_{L} p(p-1)\left\langle 1, c_{p-2}\right\rangle+\Omega(1+\gamma) p\left\langle u, c_{p-1}\right\rangle,
\end{gathered}
$$

with $p=0,1,2, \ldots$, while the boundary and initial conditions become

$$
\begin{gathered}
\frac{\partial c_{p}}{\partial r}=0 \quad \text { for } \quad r=r_{i} \quad \text { and } \quad r=1, \\
c_{p}(r, 0)=\tilde{c}_{p}(r), \quad m_{p}(0)=\left\langle x^{p}, \tilde{c}\right\rangle .
\end{gathered}
$$

By setting $p=0$ in (22), one obtains a homogeneous equation for the zeroth axial moment $c_{0}(r, t)$, solvable through separation of variables in terms of a projection onto the Fourier basis $\left\{f_{n}\right\}$, namely,

$$
c_{0}(r, t)=\sum_{n=0}^{\infty} a_{0 n} e^{-g_{r} \lambda_{n} t} f_{n}(r),
$$

where the coefficients $a_{0 n}$ satisfy the initial condition $a_{0 n}=\left\langle f_{n}, \tilde{c_{0}}\right\rangle$, while $f_{n}$ is the solution of the eigenvalue problem

$$
\left[\frac{\partial^{2}}{\partial r^{2}}+\frac{1}{r} \frac{\partial}{\partial r}+\lambda_{n}\right] f_{n}=0,\left.\quad \frac{\partial f_{n}}{\partial r}\right|_{r=1}=\left.\frac{\partial f_{n}}{\partial r}\right|_{r=r_{i}}=0
$$

The solution of the above problem is

$$
f_{n}=\frac{1}{\xi_{n}}\left[J_{0}\left(\Lambda_{n} r\right)-\frac{J_{1}\left(\Lambda_{n} r_{i}\right)}{Y_{1}\left(\Lambda_{n} r_{i}\right)} Y_{0}\left(\Lambda_{n} r\right)\right]
$$

where $\Lambda_{n}=\sqrt{\lambda_{n}}$ is the $n$th root of the characteristic equation,

$$
J_{1}\left(\Lambda_{n} r_{i}\right) Y_{1}\left(\Lambda_{n}\right)-Y_{1}\left(\Lambda_{n} r_{i}\right) J_{1}\left(\Lambda_{n}\right)=0,
$$

and $\xi_{n}$ is a factor which assures orthonormality of the solution. An example of the eigenvalue set is reported in Fig. 3. Recursively, the first axial moment is obtained by solving (22) with $p=1$ and $c_{0}(r, t)$ evaluated as above, obtaining

$$
c_{1}(r, t)=(1+\gamma) \Omega \sum_{m=0}^{\infty} \sum_{n=0}^{\infty}\left(a_{1 n} \delta_{n m}+2 a_{0 n} \operatorname{Re}\left[\beta_{m n} e^{i \omega t}\right]\right) f_{m} e^{-g_{r} \lambda_{n} t},
$$




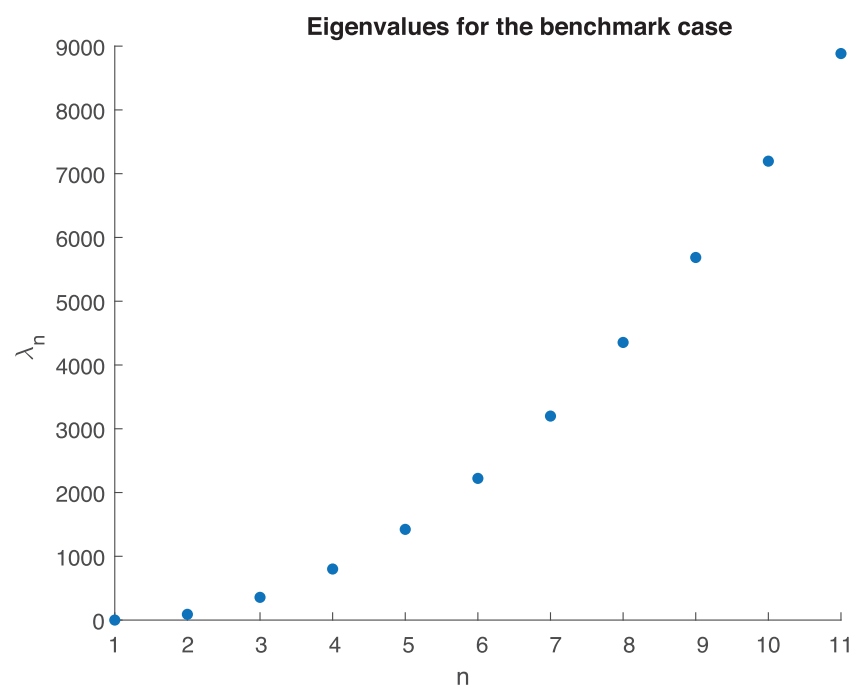

FIG. 3. Example of an eigenvalue set for the parameters reported in Table I.

with

$$
\begin{gathered}
\beta_{k n}=\frac{\left\langle f_{k} \hat{u}(r), f_{n}\right\rangle}{g_{r} \lambda_{k}-g_{r} \lambda_{n}+2 \pi i}, \\
a_{1 n}=\frac{1}{\Omega}\left\langle f_{n}, \tilde{c}_{1}(r)\right\rangle-\sum_{k=0}^{\infty} 2 a_{0 k} \operatorname{Re}\left[\beta_{n k}\right] .
\end{gathered}
$$

Let us consider the case where $\tilde{c}$ is uniformly distributed in the cross section at the initial time. In this case we obtain

$$
\begin{gathered}
m_{1}=2(1+\gamma) \Omega \operatorname{Re}\left[\langle\hat{u}, 1\rangle \frac{1-e^{2 \pi i t}}{2 \pi i}\right], \\
\frac{d m_{1}}{d t}=2(1+\gamma) \Omega \operatorname{Re}\left[\langle\hat{u}, 1\rangle e^{2 \pi i t}\right], \\
\frac{1}{2} \frac{d m_{2}}{d t}=g_{L}+(1+\gamma)^{2} \Omega^{2}\left\{\sum_{m=0}^{\infty} \sum_{l, k=-1}^{1} \frac{\left\langle\hat{u}_{k}, f_{m}\right\rangle\left\langle f_{m}, \hat{u}_{l}\right\rangle}{g_{r} \lambda_{m}+i l \omega}\left[e^{2 \pi i l t}-e^{-\lambda_{m} g_{r} t}\right] e^{-2 \pi k i t}\right\},
\end{gathered}
$$

and by integrating the last equation we get

$$
\begin{aligned}
m_{2}(t)= & g_{L} t+\Omega^{2}(1+\gamma)^{2} \sum_{n=0}^{\infty}\left\{2 \operatorname{Re}\left[\frac{\left\langle\hat{u}, f_{n}\right\rangle\left\langle f_{n}, \hat{u}\right\rangle}{g_{r} \lambda_{n}+2 \pi i}\right] t\right. \\
& +2 \operatorname{Re}\left[\frac{\left\langle\hat{u}, f_{n}\right\rangle\left\langle f_{n}, \hat{u}\right\rangle}{\left(g_{r} \lambda_{n}+2 \pi i\right)^{2}}\left(e^{-\left(\lambda_{n} g_{r}+2 \pi i\right) t}-1\right)\right]+2 \operatorname{Re}\left[\frac{\left|\left\langle\hat{u}, f_{n}\right\rangle\right|^{2}}{g_{r} \lambda_{n}+2 \pi i} \frac{e^{4 \pi i t}-1}{4 \pi i}\right] \\
& \left.+2 \operatorname{Re}\left[\frac{\left|\left\langle\hat{u}, f_{n}\right\rangle\right|^{2}}{g_{r} \lambda_{n}+2 \pi i} \frac{e^{-\left(\lambda_{n} g_{r}-2 \pi i\right) t}-1}{g_{r} \lambda_{n}-2 \pi i}\right]\right\},
\end{aligned}
$$

with $l, k \neq 0, \hat{u}_{1}=\hat{u}(r)$, and $\hat{u}_{-1}=\hat{u}^{*}(r)$, where the asterisk refers to complex conjugation. 
Finally, the (dimensionless) effective diffusivity is evaluated by substituting (5), (28), and (33)(35) into Eq. (19) and it reads

$$
D_{e}(t)=g_{L}+(1+\gamma)^{2} \Omega^{2} \sum_{n=1}^{\infty}\left\{4 \operatorname{Re}\left[\left\langle f_{n}, \hat{u}\right\rangle e^{2 \pi i t}\right] \times \operatorname{Re}\left[\frac{\left\langle f_{n}, \hat{u}\right\rangle}{g_{r} \lambda_{n}+2 \pi i}\left(e^{2 \pi i t}-e^{-g_{r} \lambda_{n} t}\right)\right]\right\} .
$$

Equation (37) is the main result of the present work, and the exact expression for $\left\langle f_{n}, \hat{u}\right\rangle$ is provided in Appendix A.

A time-scale-based analysis of the above result is instructive. In the nonporous standard case $\tau_{D L}=\tau_{D r}=\delta_{r}^{* 2} / \mathcal{D} \sim 10^{4} \tau_{\omega}$, because the lateral and longitudinal morphological diffusivities due to ligaments are both 0 [see Eqs. (14) and (15)]. Moreover, the shear time scale $\tau_{r}$ is $10^{2}$ times smaller than the oscillation time scale $\left(\tau_{\omega} \sim 1 s\right)$. Thus, since $g_{L} \ll \Omega^{2}$ and $\gamma=0$, Eq. (37) reduces to $D_{e} \sim$ $\Omega^{2} \Phi(t)$, where $\Phi(t)$ is the contribution due to oscillation given by summation in Eq. (37). When translated in dimensional units, it follows that at long term (i.e., $t \gg 1 / g_{r} \lambda_{1}$, namely, the second exponential term vanishing) the average effective diffusivity is proportional to the ratio between the square of the displacement of the tracer during the correlation time in the case of nonpulsatility and the correlation time itself,

$$
\overline{D_{e}^{*}}=\frac{\Delta_{s}^{2}}{t_{\mathrm{corr}}} \overline{\Phi^{*}}
$$

where the correlation time $t_{\text {corr }}$ is akin to the lateral diffusion time scale, i.e., $t_{\text {corr }} \sim \delta_{r}^{* 2} / \mathcal{D}$, the displacement is $\Delta_{s}=U^{*} \sqrt{\tau_{\omega} t_{\text {corr }}}$, and the overbar indicates phase-averaging.

In the presence of porosity, the transverse diffusivity $D_{r}$ is larger than the molecular diffusivity $\left(D_{r} \sim 500 \mathcal{D}\right)$ and the tortuous flow induced by ligaments reduces the time for transverse mixing to $\tau_{D r} \sim 150 \tau_{\omega}$. Moreover, in the longitudinal direction, the trabeculae induce hydrodynamic diffusion that becomes predominant in the dispersion phenomenon $\left(D_{L} \sim 2 \times 10^{4} \mathcal{D}\right)$. Thus, since $g_{L} \sim \Omega^{2}$ and $\gamma \ll 1$, Eq. (37) reduces to $D_{e} \sim g_{L}+\Omega^{2} \Phi(t)$, while the average effective diffusivity at long times in dimensional units reads

$$
\overline{D_{e}^{*}}=D_{L}+\frac{\Delta_{s}^{2}}{t_{\text {corr }}} \overline{\Phi^{*}}
$$

In addition, by following [4], we can also get a phase-averaged diffusivity, so after using Eq. (19), we obtain the solution for the time-dependent phase-averaged variance $\sigma^{2}$ of the spatial solute distribution,

$$
\begin{aligned}
\sigma^{2}(t)= & 2 \int_{0}^{t} \overline{D_{e}(t)} d t=2 \int_{0}^{t} \frac{1}{T} \int_{t}^{t+T} D_{e}\left(t^{\prime}\right) d t^{\prime} d t \\
= & g_{L} t+(1+\gamma)^{2} \Omega^{2} \sum_{n=1}^{\infty}\left\{2 \operatorname{Re}\left[\frac{\left\langle\hat{u}, f_{n}\right\rangle\left\langle f_{n}, \hat{u},\right\rangle}{g_{r} \lambda_{n}+2 \pi i}\right] t\right. \\
& \left.+4 \operatorname{Re}\left[\frac{\left\langle\hat{u}, f_{n}\right\rangle}{g_{r} \lambda_{n}+2 \pi i}\right] \times \operatorname{Re}\left[\left\langle f_{n}, \hat{u}\right\rangle \frac{1-e^{-g_{r} \lambda_{n} T}}{\left(g_{r} \lambda_{n}+2 \pi i\right)^{2}}\left(1-e^{-\left(g_{r} \lambda_{n}+2 \pi i\right) t}\right)\right]\right\} .
\end{aligned}
$$

\section{RESULTS}

\section{A. Sensitivity analysis}

A sensitive analysis is conducted here in order to investigate the impact of the SAS anatomy, the frequency of pulsatility, and the forcing pressure on the effective diffusivity, when the porous medium is neglected. In particular, values of diffusivity in the transient regime are compared. The physiological values of the parameters are chosen as a benchmark case $[8,13,16,21]$, indicated by the subscript B henceforth, and both the analytical and the numerical solutions are considered. Details of the different scenarios are reported in Table II, while details about the numerical simulations are reported in Appendix B. 
TABLE I. Physiological values of the model parameters.

\begin{tabular}{lccc}
\hline \hline Quantity & Symbol & Value & Unit \\
\hline CSF density [6,7] & $\rho$ & 1050 & $\mathrm{~kg} / \mathrm{m}^{3}$ \\
CSF dynamic viscosity [6,7] & $\mu$ & 0.001 & $\mathrm{~Pa} \mathrm{~s}$ \\
Drug molecular diffusivity & $\mathcal{D}$ & $2.1 \times 10^{-10}$ & $\mathrm{~m}^{2} / \mathrm{s}$ \\
Spinal length & $L^{*}$ & 0.50 & $\mathrm{~m}$ \\
External SAS radius [32-34] & $r_{e}^{*}$ & 0.015 & $\mathrm{~m}$ \\
Internal SAS radius [32-34] & $r_{i}^{*}$ & 0.01 & $\mathrm{~m}$ \\
Amplitude pressure wave [35] & $P_{0}$ & 250 & $\mathrm{~Pa}$ \\
Oscillation frequency [13] & $\omega$ & $2 \pi$ & $1 / \mathrm{s}$ \\
Womersley number & $W$ & 12 & \\
\hline \hline
\end{tabular}

Figure 4(a) shows that $D_{e}$ decreases moderately when the external radius of the cavity is increased (scenarios $S_{R, 1-3}$ ). This is mainly due to a decrease in the peak velocity and to the consequent reduction of the shear in the velocity profile.

The change in the pulse pressure affects the magnitude of dispersion to a great extent [see Fig. 4(b)]. For instance, a 50\% reduction in $\Delta^{*}$ causes a fourfold decrease in $D_{e}$. Although the meninges are here assumed to be undeformable and $z$ dependency of the flow field solution has been neglected, it is instructive to relate the amplitude of the pulse pressure gradient $\Delta^{*}$ to the features of real CSF flow measured in humans. As stated above, such flows are kinematic waves that normally propagate with celerity $c_{w}^{*} \sim 5-10 \mathrm{~m} / \mathrm{s}$. Basically, this celerity is related to the wavelength $l^{*}$ and the frequency according to $c_{w}^{*}=\omega^{*} l^{*} / 2 \pi$. Since $\Delta^{*}$ is the product of the pulse pressure amplitude and the spatial wave number $k^{*}$, an increase in $\Delta^{*}$ is numerically equivalent to a decrease in the wavelength $l^{*}=2 \pi / k^{*}$. Let us recall that the Moens-Korteweg equation states that the phase celerity (called the pulse-wave velocity by physicians) is proportional to the root square of the wall elastic module [36]. This means that, since the frequency is externally imposed by the heart rate, a variation in $\Delta^{*}$ can also be considered equivalent to different wall rigidities, although the present model does not account for this aspect explicitly. This aspect provides an additional interpretation of the sensitivity analysis on $\Delta^{*}$. It is in fact reasonable to suppose that more flexible boundaries may damp the dispersion of the solute within the SAS. This argument is in line with the recent analysis by Lawrence et al. [37], which modeled the dura mater (i.e., the outer wall) as an elastic membrane and did not obtain a significant role of shear dispersion. However, that analysis did not consider the role of the porous medium, an element that is shown to be significant in the next section.

Since there is a strong correlation between heart rate and CSF pulsation, an increase (decrease) in $\omega$ allows us to understand how the effective diffusivity is affected in tachycardic (brachycardic)

TABLE II. Summary of the different runs for the sensitivity analysis $\left(g_{L}=g_{r}=\tau_{\omega} / \tau_{\mathcal{D}}\right)$.

\begin{tabular}{lccc}
\hline \hline Test case & $R_{e}(\mathrm{~m})$ & $\omega^{*}(1 / \mathrm{s})$ & $L(\mathrm{~m})$ \\
\hline Benchmark & 0.015 & $2 \pi(60 \mathrm{bpm})$ & 5 \\
$S_{l, 1}$ & 0.015 & $2 \pi$ & 3 \\
$S_{l, 2}$ & 0.015 & $2 \pi$ & 8 \\
$S_{l, 3}$ & 0.015 & $2 \pi$ & 10 \\
$S_{R, 1}$ & $2 \pi$ & 5 \\
$S_{R, 2}$ & 0.0120 & $2 \pi$ & 5 \\
$S_{R, 3}$ & $2 \pi$ & 5 \\
$S_{\omega, 1}$ & 0.0135 & $11 / 6 \pi(55 \mathrm{bpm})$ & 5 \\
$S_{\omega, 2}$ & 0.0165 & $14 / 6 \pi(70 \mathrm{bpm})$ & 5 \\
$S_{\omega, 3}$ & 0.015 & $16 / 6 \pi(80 \mathrm{bpm})$ & 5 \\
\hline \hline
\end{tabular}



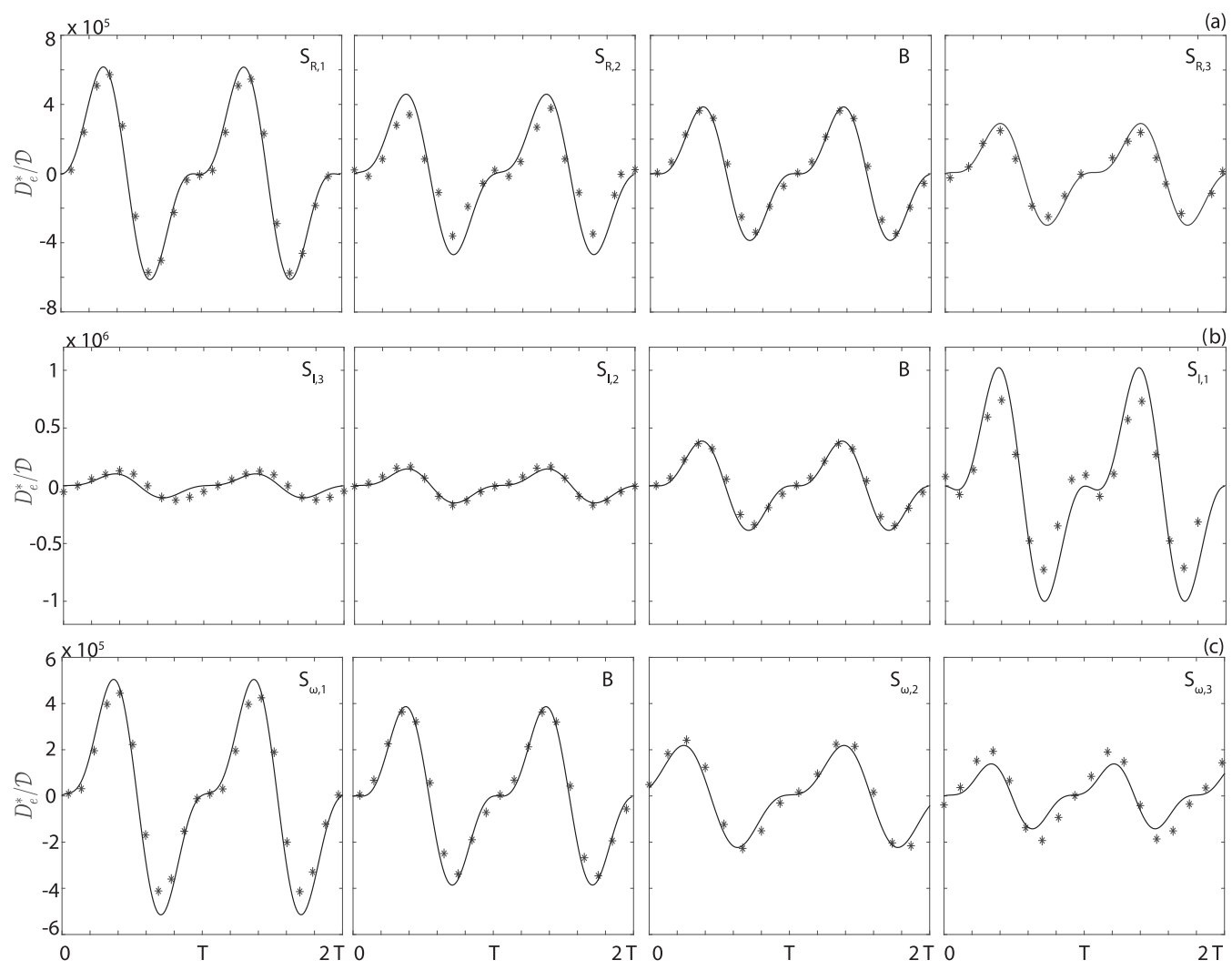

FIG. 4. Influence of the external radius $\left(S_{R, 1-3}\right)$, gradient amplitude $\left(S_{l, 1-3}\right)$, and frequency $\left(S_{\omega, 1-3}\right)$ on the effective dimensionless diffusivity. The label "B" refers to the values of the benchmark case. Solid lines refer to the analytical results, and asterisks to the numerical results (see Appendix B for further details). Note the different scaling among the rows of panels.

patients $\left(S_{\omega, 1-3}\right)$. A low value of $\omega$ (i.e., small Womersely number $W$ ) implies a well-developed velocity profile and an increase in the shear-induced dispersion [4]. Instead, if the flow varies too rapidly (high $W$ ), the velocity profile development is hindered and the peak velocity is reduced, so the shear is dampened and the dispersion of solute decreases.

We emphasize that for all tests the numerical results are in good agreement with the analytical predictions, even though the former are usually slightly higher than the latter. In addition, both numerical and analytical results are characterized by a transitory phase, with the presence of a single frequency equal to $\omega^{*}$, which gradually switches to a second harmonic $\left(2 \omega^{*}\right)$ at long times (Fig. 8). However, remember that all tests show a good agreement between analytical and numerical results after the development of the transient. For this reason, in the following the analytical solution is considered and discussed just for the time period after the end of the transitory state (but see the discussion of the key role of porosity).

\section{B. Effects of the porous medium within the SAS}

As known, obstacles in the cavity create eddies and radial motion, so enhancing dispersion [38], but also inducing additional head loss that reduces the average velocity and shear. By using the above theory, the role trabeculae play in the present problem can be assessed by observing their effects on the flow field and shear dispersion. The main features of the considered porous medium are summarized in Table III. 
TABLE III. Characteristics of the porous medium.

\begin{tabular}{lccc}
\hline \hline Quantity & Symbol & Value & Unit \\
\hline Molecular diffusivity & $\mathcal{D}$ & $2.1 \times 10^{-10}$ & $\mathrm{~m}^{2} / \mathrm{s}$ \\
SAS porosity [23,39] & $\phi$ & 0.99 & \\
Trabecular radius $[23,38]$ & $r_{T}$ & 15 & $\mu \mathrm{m}$ \\
Permeability & $\kappa$ & $1.5 \times 10^{-8}$ & $\mathrm{~m}^{2}$ \\
Transversal diffusivity & $D_{r}$ & $1.27 \times 10^{-7}$ & $\mathrm{~m}^{2} / \mathrm{s}$ \\
Longitudinal diffusivity & $D_{L}$ & $4.85 \times 10^{-6}$ & $\mathrm{~m}^{2} / \mathrm{s}$ \\
Interstitial velocity & $\hat{u}$ & 1.5 & $\mathrm{~cm}^{-}$ \\
Trabecular density & $\rho_{T}$ & 250 & $\mathrm{Tr} / \mathrm{cm}^{3}$ \\
\hline \hline
\end{tabular}

The permeability $\kappa$ is calculated through Eq. (9) by assuming standard physiological values of porosity, $\phi=0.99$, and a trabecular radius $r_{p}=15 \mu \mathrm{m}$ (Refs. [19,23,39]). We recall that the model in [25] [Eq. (11)], used here to compute the lateral $\left(D_{L}\right)$ and radial $\left(D_{r}\right)$ diffusivity, is valid for $P \gg 1$. This condition is satisfied with the physiological values of the trabecular radius $\left(\sim 15 \times 10^{-6} \mathrm{~m}\right)$, interstitial velocity $\left(\sim 2 \times 10^{-2} \mathrm{~m} / \mathrm{s}\right)$, and drug molecular diffusivity (e.g., baclofen, a common antispasticity drug administered intrathecally, has a molecular diffusivity of $\left.2.1 \times 10^{-10} \mathrm{~m}^{2} / \mathrm{s}[13]\right)$.

The trabecular pattern throughout the SAS is complex and poorly understood. The simulations in [19] considered a conservative estimate of the trabecular density $\rho_{t} \sim 85 \mathrm{Tr} \mathrm{cm}^{-3}$ in the dorsal region of the SAS, while Tangen et al. [21] analyzed a broader physiological range $\left(\rho_{t}=40\right.$ $200 \mathrm{Tr} \mathrm{cm}^{-3}$ ). Nevertheless, the impact of the trabecular density variability on the porosity is modest because of the limited volume of the single ligaments. In the present study, in agreement with the anatomical values of $\rho_{t}$, an interval of porosity from 0.999 to 0.985 has been analyzed.

Figure 5 provides a comparison of the radial distribution of velocity over the time lapse of a single period in the porous vs nonporous case. The porous medium induces a significant reduction in the maximal and mean velocity (note the different scales between the two panels) and the characteristic inflection point of the Womersley profile in the nonporous case has disappeared. In this respect, it is noteworthy that the evaluation of CSF displaced into and out of the spinal SAS during a cardiac cycle shows that the values obtained here for the porous case are more similar to MRI measurements $(\sim 2 \mathrm{ml})$ than in the nonporous case $(\sim 10 \mathrm{ml})[6,40]$.

The peak values of the dimensionless diffusivity decreases with the molecular diffusivity in a similar manner in the porous and nonporous cases [see Fig. 6(a)]. When reported in dimensional units [Fig. 6(b)], the peak diffusivity is almost independent of the molecular diffusivity when $\mathcal{D}$ is changed in the interval $10^{-11}-10^{-6} \mathrm{~m}^{2} / \mathrm{s}$. Nevertheless, the average values of diffusivity behave differently in the nonporous and porous cases (Fig. 7): when reported in dimensional units, the latter case shows $\overline{D_{e}^{*}}$ as almost independent of the molecular diffusivity, while it dramatically decreases under the nonporous condition when $\mathcal{D}$ is changed in the interval $10^{-11}-10^{-6} \mathrm{~m}^{2} / \mathrm{s}$. In other words, drug dispersion in a porous cavity is independent of the solute characteristics.

Another important result is that the fine structures within the SAS affect to a great extent the average value of $D_{e}$ (Fig. 7). This is due to the increase in $g_{L}$ and $g_{r}$ in the presence of obstacles, which spread the solute more rapidly (we recall that $g_{r}=g_{L}=\tau_{\omega} / \tau_{\mathcal{D}}$ in the nonporous case). From Eq. (37), an increase in $g_{L}$ causes an increase in the whole time series $D_{e}(t)$, while an increase in $g_{r}$ reduces the amplitude of fluctuations, so the minimum values of $D_{e}$ are always positive, and the maximum, minimum, and mean values are closer together than in the nonporous case (Fig. 8).

\section{DISCUSSION AND CONCLUSIONS}

This work provides a new modeling approach for the evaluation of dispersion of a passive scalar within an annular porous cavity under pulsatile conditions. The theoretical results have 

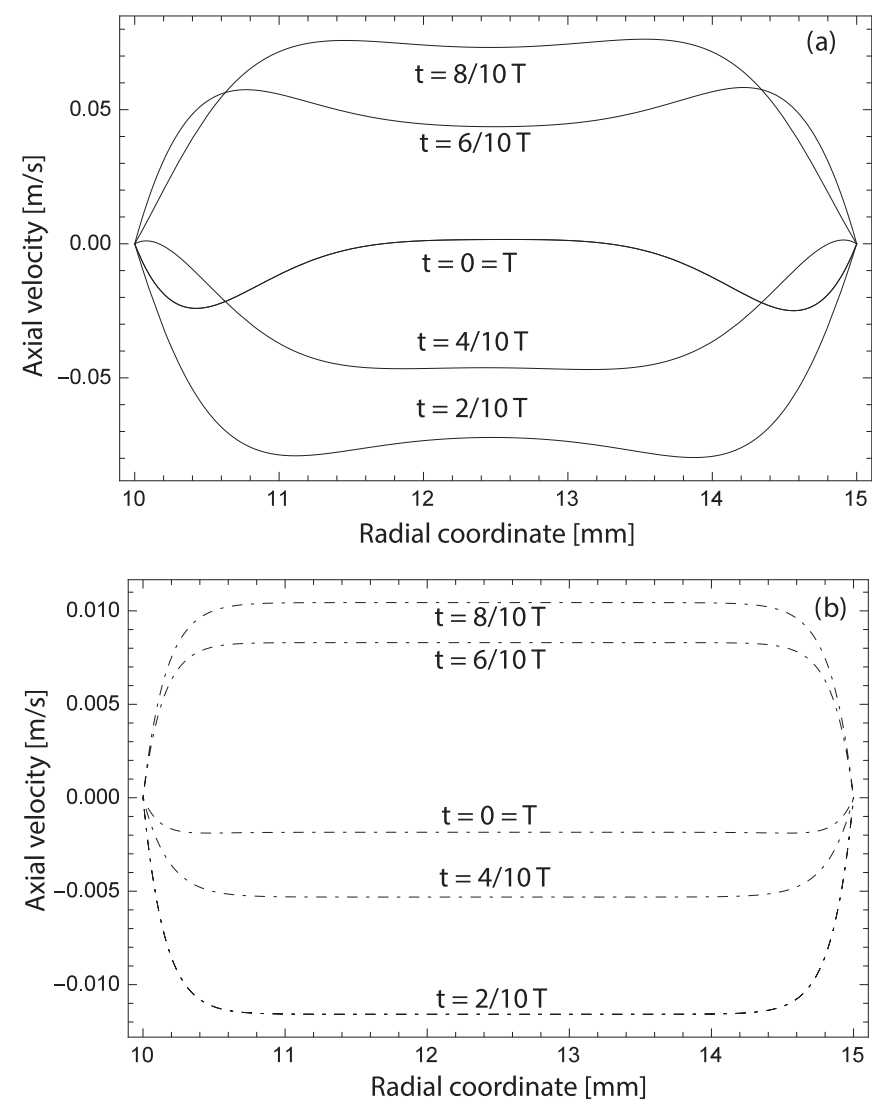

FIG. 5. Comparison of the velocity distribution between (a) the nonporous (solid curves) and (b) the porous (dot-dashed curves) cases over one time period.
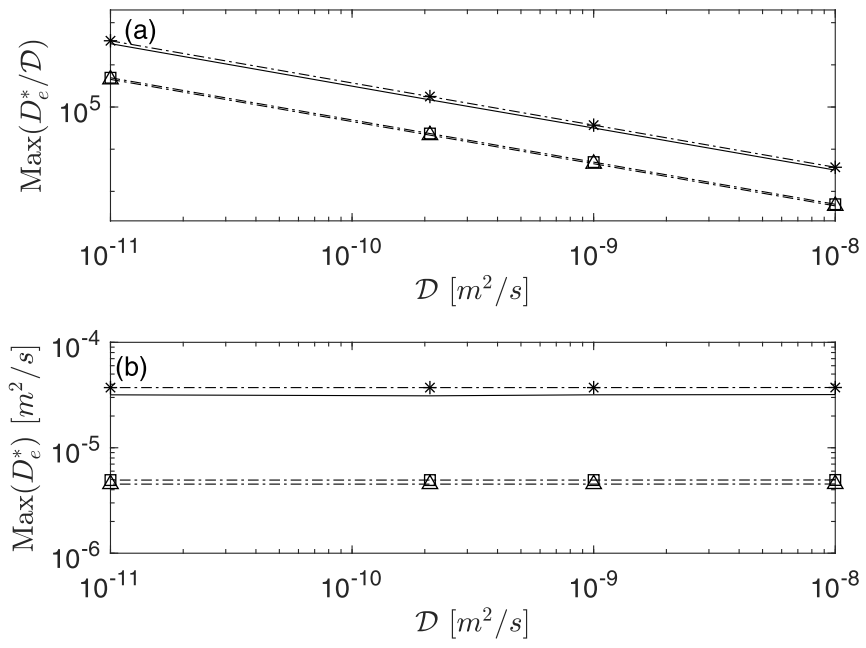

FIG. 6. Comparison of the maximum values of (a) the dimensionless vs (b) the dimensional effective diffusivity. Standard case (solid line), porous case with $\phi=0.990$ (dot-dashed line with squares), $\phi=0.999$ (dot-dashed line with asterisks), and $\phi=0.985$ (dot-dashed line with triangles). 

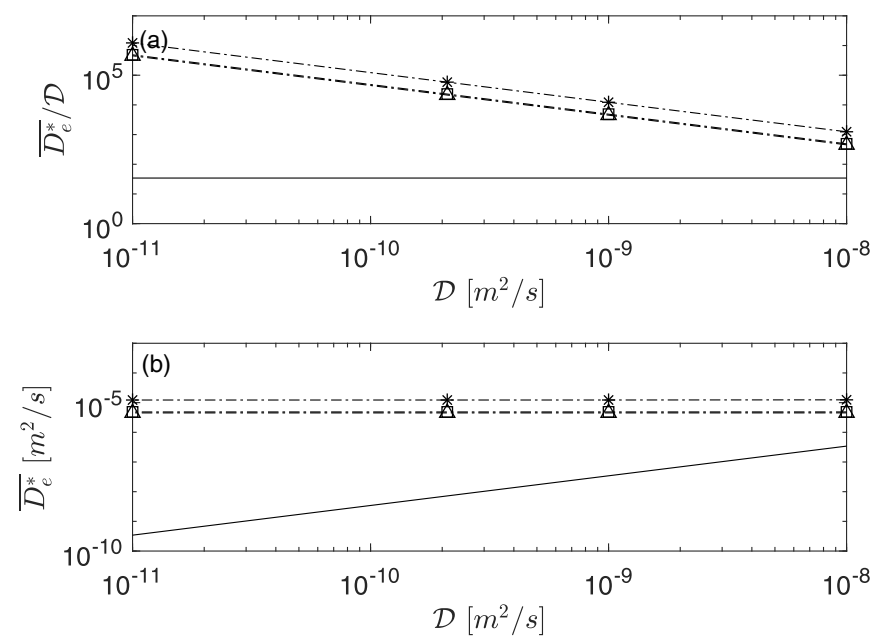

FIG. 7. Comparison of the mean values of (a) the dimensionless vs (b) the dimensional effective diffusivity. Standard case (solid line), porous case with $\phi=0.990$ (dot-dashed line with squares), $\phi=0.999$ (dot-dashed line with asterisks), and $\phi=0.985$ (dot-dashed line with triangles).

potential relevance for drug dispersion due to intrathecal injection in the cerebrospinal fluid of the subarachnoid space.

The results show that both the pulsatility and the porosity can affect the timing of drug release in a relevant way through the mechanism of shear dispersion. Due to the high value of the Womersley number $(W \sim 12)$, the velocity profile of CSF in the cavity is in fact greatly influenced by transient inertial forces, which cause a lag between the velocity and the pressure gradient and, consequently, a relatively flat distribution in the core. Moreover, the fine arachnoid ligaments, modeled here through the Brinkman term in the Navier-Stokes equations, reduce the maximal and mean velocity, cause further flattening of the core velocity and an increase in the lag between velocity and pressure (the velocity is almost in phase with $-\partial p / \partial z$ ). Nevertheless, the trabeculae

(a)
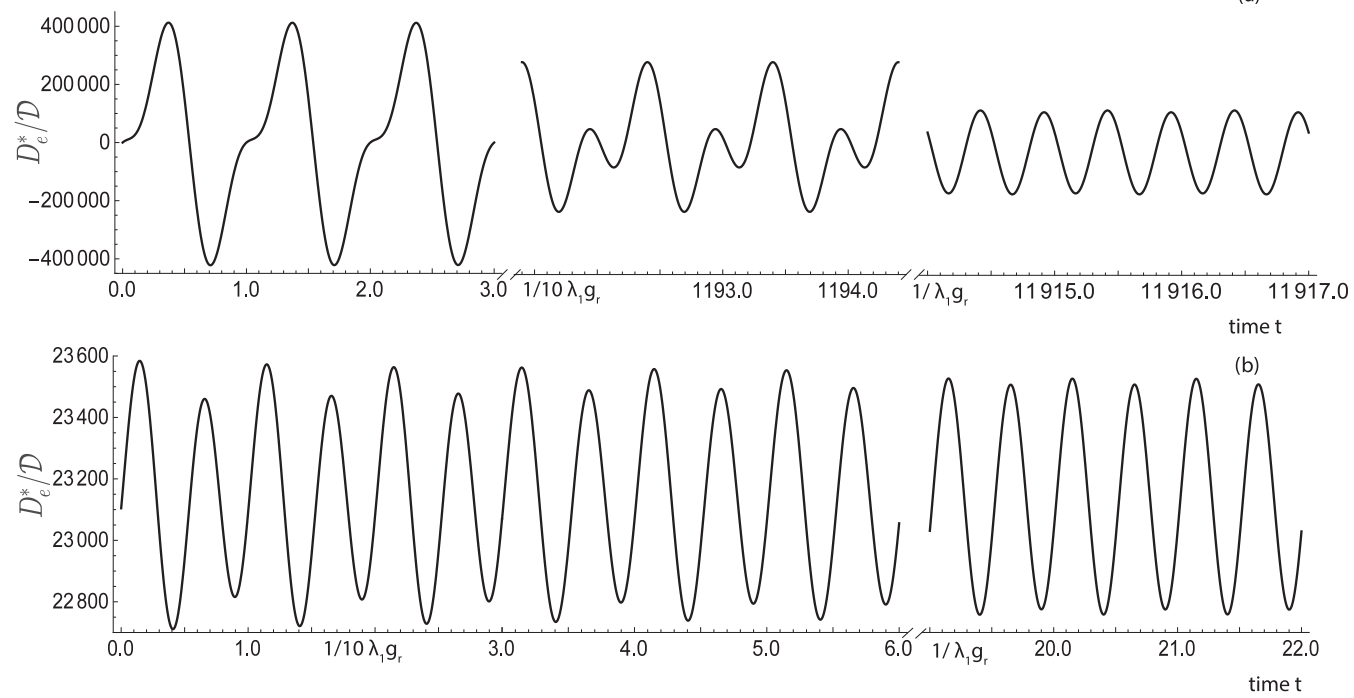

FIG. 8. Temporal behavior of the dimensionless diffusivity. (a) Nonporous case; (b) porous case. Note the differences in the scaling and in the mean values of oscillation between (a) and (b). 
produce an additional component of the diffusivity tensor (i.e., $D_{L}, D_{r}$ ) that increases the effective longitudinal diffusivity and reduces the time of solute dispersion. As a result, the characteristic time of drug dispersion, $T_{d}$, from the injection point $\left(z^{*}=0\right)$ to the cranial end $\left(z^{*}=L^{*} / 2\right)$ is between $L^{* 2} / 4 \overline{D_{e}^{*}}$ and $L^{* 2} / 4 D_{e}^{* \max }$, which is dramatically lower than the sole action of molecular diffusivity $\left(L^{* 2} / 4 \mathcal{D} \sim 10^{4} \mathrm{~h}\right)$ if the fluid is considered at rest or than the pure steady streaming advection $(\sim 4 \mathrm{~h})$ if pulsatility is neglected.

We emphasize that, although the porosity is very close to unity, the presence of ligament strands in the cavity plays a key role in further reducing $T_{d}$ with respect to the nonporous case and in weakening its dependence on the solute characteristics. In the nonporous case, $T_{d}$ is in fact highly dependent on the molecular diffusivity (spanning between 1 and $10^{6} \mathrm{~h}$ for $\mathcal{D}=10^{-6}-10^{-11} \mathrm{~m}^{2} / \mathrm{s}$ ), while in the porous case both $\overline{D_{e}^{*}}$ and $D_{e}^{* \text { max }}$ assume higher values and become closer to each other [Fig. 8(b)]. It follows that the presence of trabeculae considerably reduces its dependence on $\mathcal{D}$ and decreases the timing of dispersion (with the remarkable value of $T_{d} \sim 50 \mathrm{~min}$ for $\phi=0.999$ ). This means that the dispersion phenomenon is no longer drug specific and it develops with features that are consistent with clinical observations. Moreover, the contribution of ligaments to $D_{L}$ and $D_{r}$ increases with an increase in the porosity [25]. This means that the trabeculae affect the drug's spread even though they are present in a very limited number. With a porosity equal to 0.999 , although the maximum values of diffusivity are close to those in the nonporous case (Fig. 6), their average values are considerably higher (Fig. 7).

The sensitivity analysis showed that some features of the pressure wave, such as $\Delta$ and $\omega$, play a key role that should be carefully monitored during intrathecal treatments. In fact, tachycardic conditions (high values of $\omega$ ) or low CSF pulse pressure gradients could delay the drug delivery and the achievement of the site target of therapies. In the sensitivity analysis, mutual influence between $\omega$ and $\Delta$ was neglected, while under physiological conditions when the heart rate rises, the total blood volume inflated and deflated in each cardiac cycle changes accordingly, thus influencing the CSF pressure wave as well. To model this systemic effect could be clinically relevant but it would require coupling with a cerebral-cardiovascular simulator able to resolve the relation between $\Delta$ and $\omega$ (e.g., [41]). This aspect will be considered in a forthcoming study. On the contrary, the role of geometric parameters, such as $r_{i}$, is moderate so the approximation of a constant cross section seems quite reasonable.

Since the theoretical model does not account for a finite domain, a transient regime developed that lasted a time span $\sim\left(g_{r} \lambda_{1}\right)^{-1}$, which is much longer than the value predicted by the numerical simulations. Nevertheless, the behavior obtained by the theory after the transient is in excellent agreement with the numerical simulations. Furthermore, in the porous case, the transient regime is dramatically reduced, to only $5 \mathrm{~s}$, which is negligible compared with $T_{d}$. Therefore, the shortcoming of analyzing an infinite domain is not a real concern when porosity is considered.

Another apparent limitation of this approach is that the solute is considered to be uniformly distributed over the annular cross section at the start time. This assumption might be considered unrealistic with respect to clinical intrathecal injections where the catheter induces nonuniformity in the initial distribution. Nevertheless, the presence of trabeculae again solves the issue, since it enhances solute diffusion in the azimuthal direction, fostering the homogenization of the concentration across the annular section. The typical time of azimuthal diffusion along one-half of the annular perimeter is $\left(\pi r_{e}^{*}\right)^{2} D_{\theta}^{-1}$, where $D_{\theta}$ is the morphologically induced diffusion coefficient in the azimuthal direction. Considering the analyzed trabecular pattern (i.e., radially arranged in the SAS), we can assume that $D_{\theta}$ is between $D_{r}$ and $D_{L}$, which leads to typical time scales for the homogenization in the range $0.2-70 \mathrm{~s}$. It follows that azimuthal homogenization is obtained in a time lapse much shorter than $T_{d}$, and the assumption that the initial point distribution of the drug is uniform is quite acceptable. An exemplificative case of cloud dispersion of a drug instantaneously injected in the middle of the domain is reported in Fig. 9.

The use of Darcy's law to model anatomical fine structure could break down at the present high values of porosity. Nevertheless, this approach was tested by Gupta et al. [23] for investigating threedimensional subject-specific CSF dynamics in the inferior cranial space. Furthermore, in the present 


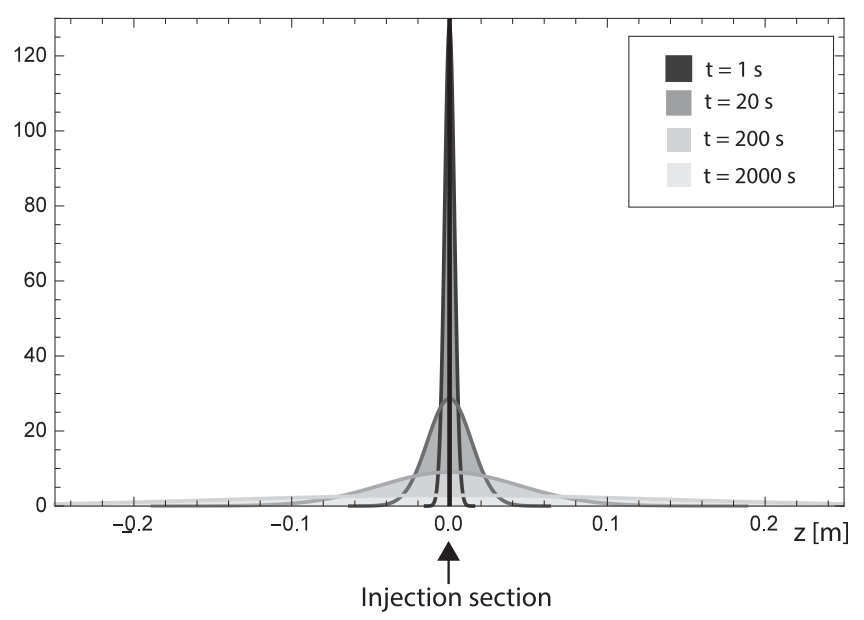

FIG. 9. Drug dispersion for an instantaneous solute injection (porous case; $\phi=0.99$ ). The spatial distribution is assumed to be Gaussian with zero mean and $\sigma^{2}$ provided by Eq. (40).

study nerve roots and dentate ligaments were neglected, but such additional fine structures within the SAS further reduce the real value of the porosity, making the Darcy assumption appropriate.

The lateral and longitudinal diffusivities derived from Koch's theory for fibrous media are basically valid in the long-time limit, namely, $t>r_{t} / U^{*} \sqrt{\phi_{r}}$. This time is less than the oscillation time, so the use of such coefficients is suitable after the first oscillation.

The actual value of the transversal hydrodynamic diffusivity $D_{r}$ should be set to 0 at the walls of the SAS, because of the no-slip condition (as discussed in [42]), instead of a constant as supposed here. This assumption generates a slight overestimation of the coefficient $g_{r}$, which affects the oscillation range of the effective diffusivity and the duration of the transitory regime. Nevertheless, the average diffusivity value remains the same and the dispersion phenomenon is unaffected [see Eq. (37)].

A recent study [37] showed that spine eccentricity may induce a steady streaming which influences drug dispersion. By considering the MRI-derived measurements of CSF flow due to production and reabsorption [7] and assuming that the whole fluid volume (cerebral and spinal) is equally involved in the circulation, the mean steady velocity of CSF in the SAS obtained is about $0.8 \mathrm{~mm} / \mathrm{min}$. This steady streaming spreads a drug bolus injected in the lumbar region throughout the SAS with a time scale $T_{d} \sim 350 \mathrm{~min}$, which is much larger than that for the pulsatility. Nevertheless, the variation of eccentricity with axial position could also induce additional secondary flows and influence transversal mixing. The issue of the combined effect of porosity and eccentricity remains open, however.

In this study, an oversimplified representation of the meningeal surface was deliberately used in order to analyze the role of pulsatile flow and the anatomical fine structure. In particular, the wall roughness was neglected. A chaotic flow triggered by the presence of ridges on the meningeal surface might generate mixing phenomena, as pointed out in [43]. However, quantification of the morphological irregularity of the SAS walls requires three-dimensional subject-specific SAS models tailored on detailed MRI [44]. A comparison between a numerical modeling of a high-resolution SAS conformation and the theoretical results, to investigate the role of wall roughness, is therefore left for future analyses.

To sum up, a complete theory able to assess longitudinal dispersion in an annular porous cavity with pulsatile flow is provided and discussed. The model has the potential torapidly and accurately assess the effective diffusivity of drugs for intrathecal therapies, highlighting the key role of the anatomical fine structures and CSF pulsations. Moreover, it allows us to identify the frequency of oscillation, the wavelength of the wave pressure, and the distance between trabeculae as the key physiological parameters of the dispersion phenomenon. 
V. NOMENCLATURE

\begin{tabular}{|c|c|}
\hline Quantity & Symbol \\
\hline Spinal subarachnoid space & SAS \\
\hline Cerebrospinal fluid & $\mathrm{CSF}$ \\
\hline Bra-ket notation & $\langle\bullet, \bullet\rangle$ \\
\hline Nondimensionless quantity & $\bullet *$ \\
\hline Cylindrical coordinate system & $(r, \theta, z)$ \\
\hline Cross section of annular cavity & $a$ \\
\hline Concentration field of solute & $c$ \\
\hline$p$ th axial moment & $c_{p}$ \\
\hline Characteristic concentration (average drug concentration value) & $C_{0}$ \\
\hline Effective diffusivity & $D_{e}$ \\
\hline Molecular diffusivity & $\mathcal{D}$ \\
\hline Longitudinal diffusivity & $D_{L}$ \\
\hline Radial diffusivity & $D_{r}$ \\
\hline$n$th basis function & $f_{n}$ \\
\hline First kind of Bessel functions of order $k$ & $J_{k}$ \\
\hline Wave number of pressure wave & $k$ \\
\hline Wavelength of pressure wave & $l$ \\
\hline Porous length scale & $l_{p}$ \\
\hline Longitudinal length of spine & $L^{*}$ \\
\hline$p$ th full moment of concentration & $m_{p}$ \\
\hline Pressure of CSF & $p$ \\
\hline Péclet number & $P$ \\
\hline Amplitude of pressure wave & $P_{0}$ \\
\hline Internal radius of SAS & $r_{e}^{*}$ \\
\hline External radius of SAS & $r_{i}^{*}$ \\
\hline Trabecular radius & $r_{t}^{*}$ \\
\hline Reynolds number & $R_{e}$ \\
\hline Schmidt number & $S$ \\
\hline Oscillation period & $T$ \\
\hline Correlation time & $t_{\text {corr }}$ \\
\hline Axial velocity component of CSF & $u$ \\
\hline Interstitial velocity & $\hat{u}_{p}$ \\
\hline Second kind of Bessel functions of order $k$ & $Y_{k}$ \\
\hline Womersley number & $W$ \\
\hline Porous Womersley number & $W_{p}$ \\
\hline Generalized wave number & $\alpha$ \\
\hline Kronecker delta & $\delta_{m, n}$ \\
\hline Amplitude of pressure-gradient wave & $\Delta$ \\
\hline CSF density & $\rho$ \\
\hline CSF viscosity & $\mu$ \\
\hline Permeability of SAS (porous medium) & $\kappa$ \\
\hline Spatial variance of solute concentration & $\sigma^{2}$ \\
\hline Porosity of SAS & $\phi$ \\
\hline Shear time scale & $\tau_{r}$ \\
\hline Diffusive time scale & $\tau_{\mathcal{D}}$ \\
\hline Lateral diffusion time scale & $\tau_{D L}$ \\
\hline Transversal diffusion time scale & $\tau_{D r}$ \\
\hline Oscillating time scale & $\tau_{\omega}$ \\
\hline Frequency of CSF oscillation & $\omega$ \\
\hline
\end{tabular}




\section{APPENDIX A: ANALYTICAL RESULTS OF INTEGRALS}

The general form of the integrals in (5) reads

$$
\begin{aligned}
\left\langle f_{n}, \hat{u}\right\rangle= & \frac{2 u_{0}}{\xi_{n} \Lambda_{n}\left(r_{i}^{2}-1\right)\left(\Lambda_{n}^{2}-\alpha^{2}\right) Y_{1}^{n}}\left[\frac{2 b_{1} \alpha J_{1}^{r}}{\pi}-\frac{2 b_{2} \alpha Y_{1}^{r}}{\pi}+\left(\alpha^{2}-\Lambda_{n}^{2}\right) J_{1}^{\Lambda} Y_{1}^{n}\right. \\
& +J_{1}^{n}\left(b_{2} \alpha \Lambda_{n} Y_{0}^{\Lambda} Y_{1}^{\alpha}+Y_{1}^{\Lambda}\left(\alpha^{2}+\Lambda_{n}^{2}\left(b_{1} J_{0}^{\alpha}-b_{2} Y_{0}^{\alpha}-1\right)\right)\right) \\
& -b_{1} \Lambda_{n}^{2} J_{0}^{\alpha} J_{1}^{\Lambda} Y_{1}^{n}+b_{2} \Lambda_{n}^{2} J_{1}^{\Lambda} Y_{0}^{\Lambda} Y_{1}^{n} \\
& \left.-b_{2} \alpha \Lambda_{n} J_{0}^{\Lambda} Y_{1}^{\Lambda} Y_{1}^{n}+b_{1} \alpha \Lambda_{n} J_{1}^{\alpha}\left(-J_{1}^{n} Y_{0}^{\Lambda}+J_{0}^{\Lambda} Y_{1}^{n}\right)\right]
\end{aligned}
$$

where

$$
\begin{gathered}
Y_{i}^{r}=Y_{i}\left[r_{i} \alpha\right], \quad J_{i}^{r}=J_{i}\left[r_{i} \alpha\right], \quad Y_{i}^{n}=Y_{i}\left[r_{i} \Lambda_{n}\right], \quad J_{i}^{n}=J_{i}\left[r_{i} \Lambda_{n}\right] \\
Y_{i}^{\Lambda}=Y_{i}\left[\left(1+r_{i}\right) \Lambda_{n}\right], \quad J_{i}^{\Lambda}=J_{i}\left[\left(1+r_{i}\right) \Lambda_{n}\right], \quad Y_{i}^{\alpha}=Y_{i}\left[\left(1+r_{i}\right) \alpha\right], \quad J_{i}^{\alpha}=J_{i}\left[\left(1+r_{i}\right) \alpha\right] .
\end{gathered}
$$

\section{APPENDIX B: NUMERICS}

A numerical validation of the theoretical solution was developed through a comparison with CFD runs using the software Comsol 4.2a. In agreement with the theoretical framework, the computational domain is an annular cavity bounded by two concentric cylindrical surfaces, composed of the upper and the lower parts of the SAS, with a thin ring for the injection of the solute in between. In order to reduce computational times, we have taken advantage of the azimuthal symmetry, thus considering a two-dimensional axisymmetric model. The domain was spatially discretized by $5 \times 10^{4}$ triangular elements. The solver resolves the continuity equation, Navier-Stokes equations, and advection-diffusion equation and computes the flow field and the concentration field in the SAS. The simulations were conducted under unsteady conditions with a time step equal to $0.05 \mathrm{~s}$. According to Eq. (1), at the inlet (cranial end of the domain), a time-dependent driving pressure was imposed,

$$
P_{\text {inlet }}=P_{0} \cos \left(\omega^{*} t^{*}\right),
$$

where $P_{0}$ is the steady pressure of the CSF within the SAS. In order to numerically emulate the propagation of a wave in a rigid domain, at the outlet boundary (the caudal end), the lagged timedependent pressure was set:

$$
P_{\text {outlet }}=P_{0} \cos \left(\omega^{*} t^{*}-k^{*} L^{*}\right) .
$$

This trick was adopted in order to numerically mimic the effect of gradient oscillation-as it was considered in the theory-without embarking on the numerical simulation of a compressible fluid in a deformable domain. Figure 10 reports the pressure field and its $z$ derivative - the pressure gradient - along the tube at different times, as resolved by the numerical simulation. It is evident that the pressure gradient is practically constant throughout the domain, an aspect that justifies the assumptions of the present theory [namely, Eq. (1)].

The convection-diffusion equation was solved by considering the injected drug as a passive scalar [17] and the solute concentration was set equal to $\tilde{c}=1$ at the injection, while at the inlet and outlet boundaries zero concentration was imposed in order to mimic no leakage of the drug from the SAS. To avoid numerical errors, a smoothed step function was also applied to the initial condition. Finally, zero concentration flux and no slip condition were imposed at the walls.

Figure 1 shows how the tracer profile reflects the velocity profile at different times during the time span of a single period $T$. The two horizontal lines refer to the advance of the front position of the same isoconcentration contour line after a single period. From the numerical solution of 

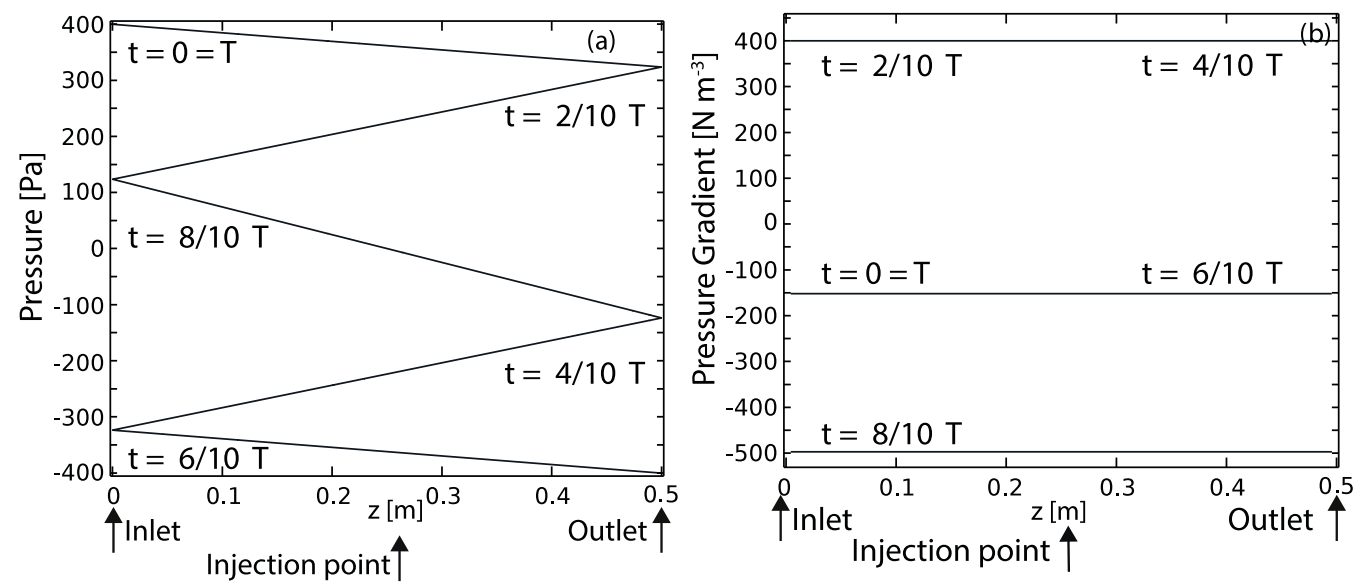

FIG. 10. Numerical solution for (a) the pressure field and (b) the pressure gradient along the tube, over one time period.

the concentration field, we spatially integrated the concentration fields at different time steps and computed $M_{1}(t), d M_{1} / d t$, and $d M_{2} / d t$. According to (19), we finally get the value of $D_{e}(t)$.

Table I summarizes the physiological values that have been considered as the benchmark case for the numerical validation and the sensitivity analysis.

[1] G. Taylor, Dispersion of soluble matter in solvent flowing slowly through a tube, Proc. R. Soc. A 219, 186 (1953).

[2] R. Aris, On the dispersion of a solute in a fluid flowing through a tube, Proc. R. Soc. A 235, 67 (1956).

[3] E. Watson, Diffusion in oscillatory pipe flow, J. Fluid Mech. 133, 233 (1983).

[4] S. Vedel and H. Bruus, Transient Taylor-Aris dispersion for time-dependent flows in straight channels, J. Fluid Mech. 691, 95 (2012).

[5] S. Vedel, E. Hovad, and H. Bruus, Time-dependent Taylor-Aris dispersion of an initial point concentration, J. Fluid Mech. 752, 107 (2014).

[6] A. A. Linninger, K. Tangen, C.-Y. Hsu, and D. Frim, Cerebrospinal fluid mechanics and its coupling to cerebrovascular dynamics, Annu. Rev. Fluid Mech. 48, 219 (2016).

[7] F. Loth, M. A. Yardimci, and N. Alperin, Hydrodynamic modeling of cerebrospinal fluid motion within the spinal cavity, J. Biomech. Eng. 123, 71 (2001).

[8] E. R. Kandel, J. H. Schwartz, T. M. Jessell, S. A. Siegelbaum, and A. J. Hudspeth, Principles of Neural Science, Vol. 4 (McGraw-Hill, New York, 2000).

[9] S. Belverud, A. Mogilner, and M. Schulder, Intrathecal pumps, Neurotherapeutics 5, 114 (2008).

[10] R. M. Nelissen, Fluid mechanics of intrathecal drug felivery, Ph.D. thesis, Swiss Federal Institute of Technology Lausanne, 2008.

[11] Y. Lee, C. Hsieh, J. Chuang, and C. Li, The necessity of intrathecal chemotherapy for the treatment of breast cancer patients with leptomeningeal metastasis: A systematic review and pooled analysis, Curr. Probl. Cancer 41, 355 (2017).

[12] F. Remes, R. Tomas, V. Jindrak, V. Vanis, and M. Setlik, Intraventricular and lumbar intrathecal administration of antibiotics in postneurosurgical patients with meningitis and/or ventriculitis in a serious clinical state, J. Neurosurg. 119, 1596 (2013).

[13] H. Hettiarachchi, Y. Hsu, T. J. Harris, and A. A. Linninger, The effect of pulsatile flow on intrathecal drug delivery in the spinal canal, Ann. Biomed. Eng. 39, 2592 (2011). 
[14] M. Wallace and T. Yaksh, Characteristics of distribution of morphine and metabolites in cerebrospinal fluid and plasma with chronic intrathecal morphine infusion in humans, Anesth. Analg. 115, 797 (2012).

[15] E. Buchser, A. Durrer, D. Chédel, and J.-P. Mustaki, Efficacy of intrathecal bupivacaine: How important is the flow rate? Pain Med. 5, 248 (2004).

[16] Y. Hsu, H. M. Hettiarachchi, D. C. Zhu, and A. A. Linninger, The frequency and magnitude of cerebrospinal fluid pulsations influence intrathecal drug distribution: Key factors for interpatient variability, Anesth. Analg. 115, 386 (2012).

[17] P. T. Haga, G. Pizzichelli, M. Mortensen, M. Kuchta, S. H. Pahlavian, E. Sinibaldi, B. A. Martin, and K.-A. Mardal, A numerical investigation of intrathecal isobaric drug dispersion within the cervical subarachnoid space, PloS One 12, e0173680 (2017).

[18] G. Pizzichelli, B. Kehlet, Ø. Evju, B. Martin, M. Rognes, K. Mardal, and E. Sinibaldi, Numerical study of intrathecal drug delivery to a permeable spinal cord: Effect of catheter position and angle, Comput. Methods Biomech. Biomed. Eng. Imaging Vis. 20, 1599 (2017).

[19] H. W. Stockman, Effect of anatomical fine structure on the dispersion of solutes in the spinal subarachnoid space, J. Biomech. Eng. 129, 666 (2007).

[20] N. Alperin, E. M. Vikingstad, B. Gomez-Anson, and D. N. Levin, Hemodynamically independent analysis of cerebrospinal fluid and brain motion observed with dynamic phase contrast MRI, Magn. Reson. Me. 35, 741 (1996).

[21] K. M. Tangen, Y. Hsu, D. C. Zhu, and A. A. Linninger, CNS wide simulation of flow resistance and drug transport due to spinal microanatomy, J. Biomech. 48, 2144 (2015).

[22] B. Sweetman and A. A. Linninger, Cerebrospinal fluid flow dynamics in the central nervous system, Ann. Biomed. Eng. 39, 484 (2011).

[23] S. Gupta, M. Soellinger, P. Boesiger, D. Poulikakos, and V. Kurtcuoglu, Three-dimensional computational modeling of subject-specific cerebrospinal fluid flow in the subarachnoid space, J. Biomech. Eng. 131, 021010 (2009).

[24] C. Camporeale, E. Mantelli, and C. Manes, Interplay among unstable modes in films over permeable walls, J. Fluid Mech. 719, 527 (2013).

[25] D. Koch and J. Brady, The effective diffusivity of fibrous media, AIChE J. 32, 575 (1986).

[26] L. Spielman and S. L. Goren, Model for predicting pressure drop and filtration efficiency in fibrous media, Environ. Sci. Technol. 2, 279 (1968).

[27] J. Westhuizen and J. P. D. Plessis, Quantification of unidirectional fiber bed permeability, J. Compos. Mater. 28, 619 (1994).

[28] P. Saffman, Dispersion due to molecular diffusion and macroscopic mixing in flow through a network of capillaries, J. Fluid Mech. 7, 194 (1960).

[29] E. J. List and N. H. Brooks, Lateral dispersion in saturated porous media, J. Geophys. Res. 72, 2531 (1967).

[30] Y. Tanino and H. M. Nepf, Laboratory investigation of lateral dispersion within dense arrays of randomly distributed cylinders at transitional reynolds number, Phys. Fluids 21, 046603 (2009).

[31] N. Barton, On the method of moments for solute dispersion, J. Fluid Mech. 126, 205 (1983).

[32] L. Ros, J. Mota, A. Guedea, and D. Bidgood, Quantitative measurements of the spinal cord and canal by MR imaging and myelography, Eur. Radiol. 8, 966 (1998).

[33] T. Kameyama, Y. Hashizume, and G. Sobue, Morphologic features of the normal human cadaveric spinal cord, Spine 21, 1285 (1996).

[34] H. Ko, J. Park, Y. Shin, and S. Baek, Gross quantitative measurements of spinal cord segments in human, Spinal Cord 42, 35 (2004).

[35] A. A. Linninger, M. Xenos, B. Sweetman, S. Ponkshe, X. Guo, and R. Penn, A mathematical model of blood, cerebrospinal fluid and brain dynamics, J. Math. Biol. 59, 729 (2009).

[36] W. Nichols and M. O'Rourke, McDonald's Blood Flow in Arteries, 5th ed. (Hodder Arnold, Devon, UK, 2005).

[37] J. Lawrence, W. Coenen, A. Sanchez, G. Pawlak, C. Martinez-Bazan, V. Haughton, and J. Lasheras, On the dispersion of a drug delivered intrathecally in the spinal canal, J. Fluid Mech. 861, 679 (2019). 
[38] H. W. Stockman, Effect of anatomical fine structure on the flow of cerebrospinal fluid in the spinal subarachnoid space, J. Biomech. Eng. 128, 106 (2006).

[39] Y. Tada and T. Nagashima, Modeling and simulation of brain lesions by the finite-element method, IEEE Eng. Med. Biol. Mag. 13, 497 (1994).

[40] M. Freund, M. Adwan, H. Kooijman, S. Heiland, M. Thomsen, S. Hähnel, K. Jensen, H. Gerner, and K. Sartor, Measurement of csf flow in the spinal canal using MRI with an optimized MRI protocol: Experimental and clinical studies, RöFo 173, 306 (2001).

[41] A. Guala, C. Camporeale, F. Tosello, C. Canuto, and L. Ridolfi, Modelling and subject-specific validation of the heart-arterial tree system, Ann. Biomed. Eng. 43, 222 (2014).

[42] D. L. Koch, Hydrodynamic diffusion near solid boundaries with applications to heat and mass transport into sheared suspensions and fixed-fibre beds, J. Fluid Mech. 318, 31 (1996).

[43] A. D. Stroock, S. K. Dertinger, A. Ajdari, I. Mezić, H. A. Stone, and G. M. Whitesides, Chaotic mixer for microchannels, Science 295, 647 (2002).

[44] L. R. Sass, M. Khani, G. C. Natividad, R. S. Tubbs, O. Baledent, and B. A. Martin, A 3D subject-specific model of the spinal subarachnoid space with anatomically realistic ventral and dorsal spinal cord nerve rootlets, Fluids Barriers CNS 14, 36 (2017). 